\title{
Oral Ulcerations
}

\author{
Magdy K Hamam ${ }^{1}$, Hamad N. Albagieh ${ }^{2}$, AL dosari AM $^{3}$ \\ ${ }^{1}$ Professor \& Head Division of Oral Medicine, \\ ${ }^{2}$ Assistant Professor, Chairman, Department of Oral Medicine \& Diagnostic Sciences \\ ${ }^{3}$ Professorof Oral Medicine , Former Dean, College of Dentistry, King Saud University, Saudi Arabia
}

Received: January 08, 2021; Accepted: January 23, 2021; Published: February 02, 2021

“Corresponding author: Magdy K Hamam, Professor \& Head Division of Oral Medicine, E-mail: mkkhaled58@hotmail.com

\section{Introduction}

The Oral cavity is a mirror of systemic conditions.

Many diseases have a similar clinical appearance, so a dentist attempting to diagnose an ulcerative or vesiculobullous disease of the mouth needs a good experience. The oral mucosa is thin, causing vesicles and bullae to break rapidly into ulcers, and ulcers are easily traumatized from teeth and food, and they become secondarily infected by the oral flora. These factors may cause lesions that have a characteristic appearance on the skin to have a nonspecific appearance on the oral mucosa. Oral manifestations may precede or follow the appearance of findings at other locations. A complete system review should be obtained a brief history and rapid clinical examination and full investigations for each patient, including questions regarding the presence of skin, eye, genital, and rectal lesions. As well as symptoms such as joint pains, muscle weakness, dyspnea, diplopia, and chest pains $[1,2]$.

So, the present review article will include, Definitions, Classifications ,Clinical features, Pathogenesis, Diferetional diagnosis , Treatment \& new trends .

Oral ulcers may be classified to Primary or Secondary ulcers

1- Primary Ulcers which are not preceded by vesiculo-bullous lesion :- for example :-

a-Traumatic

b- Infective : Bacterial \& fungal infection

c- Neoplastic

d-Systemic : GIT \& blood disorders

e- Aphthous

f- Behcet 'syndrome

h- Reiter 's syndrome

2- Secondary ulcers: which are preceded by vesiculo-bullous lesion .Vesicles may be either:-

\section{A- Intraepithelial or sub-epithelial}

- $\quad$ Primary Herpetic gingivostomatitis.
- Recurrent Herpes labialis.

- Herpes zoster.

- Herpangina.

- Hand, foot \& mouth disease.

- Pemphigus vulgaris.

B-Sub- Epithelial Vesiculobullous Lesions

Bullous Pemphigoid

Benign mucous membrane Pemphigoid

Erosive Lichen Planus

Erythema Multiforme

EpidermolysisBullosa

Dermatitis Herpetiformis

\section{Recurrent Herpes Simplex Virus infection}

\section{A- Primary Herpetic Gingivostomatitis infection}

B- Recurrent Herpes Simplex infection.It contains DNA acid nucleus.

It can remain latent in host neural cells there by evading the host immune response

- A-HSV1 ( above the waist)

- Oral \& pharyngeal infection

- Meningoencephalitis

- Dermatitis above waist .

- B- HSV2 ( under the waist)

- Genital infections

- Infection in new born

- Dermatitis below waist rus infection

- Recurrent Herpes Simplex Virus infection

- a- Primary Herpetic gingivstomatitis 
- Prodromal symptoms. ( before oral lesions )

1, 2days $\rightarrow$ Fever, headache, malaise, nausea, vomiting $\rightarrow 2-3$ days $\rightarrow$ Lesions $\rightarrow$ 7-10 days $\rightarrow$ self-limiting

HSV may continue to be present in the saliva for up to month after the onset disease.

So, it can contaminate with injury of dentist finger to presented as Primary herpetic Whitlow on the finger of a dentist. Ocular herpes simplex virus type 1 infection from rubbing the eye with a saliva-contaminated finger in a patient with primary herpetic stomatitis

\section{b- Recurrent Herpes Simplex Virus infection}

- RHS is not a re-infection but re-activation of

- Virus that remain latent in nerve tissue in a non-replicating state.

- RHS may be activated by:

- Trauma ,fever, sunburn, immunosuppression, menstruation

- The virus travels down the nerve trunk to infect epithelial cells

- Spreading from cell to cell cause a lesion

Predisposing factors

- Fever, menstruation, ultraviolet emotional stress

- Prodromal

- Tingling or burning

- Formation of a cluster of small vesicles, each

- Vesicle 1-3 mm in diameter with the size of the

- Cluster ranging from 1-2 cm.

- Vesicles rupture $\rightarrow$ ulcer

- Site :- (recurrent intra oral herpes-, gingiva, palate alveolar ridge (kerat.mucosa)

\section{Diagnosis}

Case history, clinical examination \& special investigations

1- Cytology

2- HSV isolation (most positive method)

Isolation \& neutralization of a virus in tissue culture.

3- Antibody Titters.

A convalescent serum can confirm the diagnosis of primary HSV infection by demonstrating at

Least a fourfold rise in anti-HSV antibody
Laboratory diagnosis

- 1- cytology

- Fresh vesicle \& stained by Giemsa,Wright's

- Or Papanicolaou's stain

- Searching for multinucleated giant cells

- Ballooning degeneration of nucleus

- Fluorescent staining of cytology smear (sensitive).

Treatment

1- Acyclovir

- No effect on normal cells but inhibits DNA replication in HSVinfected cells.

- It is effective in first 72 hours

- New antiherpes drugs are now available :-

- Val acyclovir\&Famciclovir $\rightarrow \rightarrow$ it increased bioavailability allowing for effective treatment with fewer dose..

- Analgesic \& Topical anasethia. ( mild case )

2- Antibiotics are of no help in treatment of primary herpes infection.

3- Corticosteroid is contraindicated

4- Vaccine (in future; geno-therapy).

\section{Varicella - Zoster Virus infection}

- It is a herpes virus.

- It causes primary \& recurrent infection \& remains latent in neurons present in sensory ganglia.

- VZV is responsible for 2 major clinical infection :-

- Of humans Chic hen Pox (varicella )( Primary infection)

- After the primary disease is healed,VZV become latent in the dorsal root ganglia of spinal nerves or extramedully ganglia of cranial nerves

- Shingles ( Herpes Zoster) Varicella - Zoster Virus infection ( secondary infection )

- Vesicle are unilateral \& accompanied by pain along the course of one trigeminal nerve branch.

Skin lesions

- Painful , unilateral, shallow, small, round with erythematous base, healing by scar .

- N.B. Child without prior contact with VZV canDevelop chicken pox after contact with an individual with $\mathrm{HZ}$. 
Complications

- Post herpetic neuralgia ; pain remaining for over a month after the mucocutaneous lesions have healed

- Healing :- within 3-4 weeks by scar formation

The risk increases after the age of 60 years due to the decline in cell- mediated immunity.

- Immunosuppression does not increase the risk of post herpetic neuralgia .

Treatment

- To prevent post herpetic neuralgia in elder patient.

- Dissemination in immunocompromisedpatient.

- Acyclovir

- Antiherpes drugs ( valacyclovir - famciclovir) accelerate healing and reduce acute pain but they do not reduce the incidence of post herpetic neuralgia

- Capsaicin( sub extracted from hot chili peppers)

- Chemical or surgical neurolysis done in refractory cases.

\section{Ramsay Hunt syndrome}

- Definition:- It is special-form of HZ affecting the facial nerve via infection of geniculate ganglion.

- HerpaticOticus :- small crops of vesicles ( tragus of ear , extra auditory canal, tympanic membrane )

- Oral manifestations

- Unilateral localized oral pain affecting the anterior two thirds of the tongue, soft palate followed by vesicular lesions $\rightarrow$ ulcers

- Bell ‘s Palsy

- Loss of taste sensation

- Complications :- course is rapid resolution within 7-10 days

Deafness - permanent paralysis

- Treatment:

- Prednisone $60 \mathrm{mg}$ daily reduced to nil over $10 \mathrm{~d}$

\section{Acute Necrotizing Ulcerative Gingivitis(ANUG )}

It is an endogenous oral infection that is characterized by necrosis of the gingiva

- $\quad$ Trench mouth

- Overgrowth of normal oral flora \& is not transmissible

- $\quad$ Etiology :-

1- Fusiform bacillus \& Spirochetes.(endotoxins act directly on tissues or indirectly by triggering immunologic \& inflammatory reactions $\rightarrow$ tissue destruction)

- 2-Poor oral hygiene, faulty dental restorations.

- 3- Smoking

- 4- Emotional stress.

- 5- Leukemia \& Aplastic anemia (defect of neutrophils)6Malnutrition \& HIV infection

\section{Secondary Tuberculosis ( T. B .)}

Etiology :-Mycobacterium tuberculosisinfection.

Chronic, indurate ulcer, irregular, undermined edge

Thick mucus material in the base

Painless, without lymphadenitis

\section{4-Cyclic Neutropenia}

- Etiology A hereditary autosomal dominant trait has been recorded insome cases.

- Clinical features The disease is usually manifested in childhood, and

- The reduction of neutrophils occurs regularly in a 21-day cycle.

- Patients,typically may complain of low-grade fever, headache, malaise, anorexia,

- arthralgias, cervical lymphadenopathy, gastrointestinal disorders,.

- Oral lesions present as a painful ulcer

- covered by a whitish membrane and surrounded by an erythematoushalo .Localized gingivitis is also a common finding.

- Laboratory tests Determination of neutrophils in the peripheral blood

(usually two or three times per week for eight weeks).

\section{Agranulocytosis}

DefinitionAgranulocytosis is a hematological disorder characterizedby a severe reduction of the granulocyte series, particularly neutrophils

- Etiology Drugs or infections are commonly the cause, although some

- cases are idiopathic

- Clinical features The disease has a sudden onset and is characterized

- by chills, fever, malaise, and sore throat. Bacterial infections 
often develop.

- Oral lesions are common early signs, and consist of multiple necrotic

- ulcers covered by a grayish-white or dark and dirty pseudo membrane

- ithout a red haloThe buccal mucosa, tongue, palate, and

- tonsillar area are the most common sites of involvement. Severe necrotizing gingivitis with periodontal tissue destruction is common.

- The systemic manifestations include sudden chills, fever, malaise, and

- sore throat. Within 12-24 hours, signs and symptoms of respiratory and/ or gastrointestinal tract or other bacterial infections may develop.

- The risk of infections is usually related to the degree of neutropenia.

- Laboratory tests White blood count and bone-marrow aspiration.

\section{Recurrent Aphthous Stomatitis (RAS)}

- It is a disorder characterized by recurring ulcers confined to oral mucosa

- With no other signs of disease.

- Etiology :-

-1- Immunologic \& genetically disorders.

-2-Hematologic deficiencies.

- 3- Psych. Abnormalities, upper respiratory infection

- 4-Trauma, anxiety, stress, menstruation.

- 5- Refractory disease; allergy to food (milk, cheese, wheat, flour toothpaste.)

\section{Classification:}

- 1- Major ulcers (Sutton's disease; peri adenitis mucosa necrotic a recurrent)

- 2-Minor ulcers

- 3- Herpetiformis

\section{Clinical manifestation}

-1- Prodromal burning sensation (2-48hours).

-2- localized area of erythema (within hours )
- 3-Smal white papule forms, ulcerates (48-72 h.)

- Site :-

- Non- keratinized mucosa is common than keratinized mucosa.

- Differences between minor and major aphthous ulcers

\section{Diagnosis:}

- By exclusion

- Differential diagnosis

- History :\& examination

-1-Primary acute lesions( viral stomatitis )

-2-Chronic multiple lesions(Pemphigoid)

-3-Recurring ulcers (dermal disorder)

- Laboratory investigation(worsen ulcer )

- Biopsies (only to exclude other diseases; Crohn's disease or sarcoidosis. )

- Systemic conditions associated withAphthouslike ulceration:-

* Behcet's Syndrome

* Reiter's Syndrome

* Cyclic neutropenia

* Agranulocytosis

HIV

\section{MucoCutaneous- Occular Syndrome}

- A- Steven Johnson's Syndrome

- B- Behcet's Syndrome

- C- Reiter's Syndrome

\section{7-A Steven Johnsons ‘s Syndrome}

(Mucocutaneous-ocularsyndrome; major form)

It is characterized by generalized vesiculo-bullous lesions involving the skin, mouth, eyes \& genitalia .

Age :- infants , children \& young adults .sex :- both sexes

Onset: - fever , headache , malaise , anorexia sever vesiculobullous lesions within 24-48 hours

Fever may persist 4-5 days, \& after 14 days patient appears prostrated,toxic, dehydrated \&actually ill

Oral lesions: 
It is inflammatory \& vesicular lesions.

Vesicles develop on erythematous base $\rightarrow$ ulceration.

Lips: - vermillion border gets covered by bloody crusted lesion

Tongue: - enlarged with indentation \& erosion on anterior \& lateral border.

Symptoms: - pain, discomfort, inability to eat, swallowing difficulty increased salivation.

\section{Skin lesion:-}

Red or violaceous maculae or papules on back of hands, forearms , feet \& legs ,knees \& side of neck iris or target lesions , concentric erythematous ring on hands \& wrists .

- vesiculo-bullouslesions skin sloughing $\rightarrow$ death ( due to secondary infection \& or fluid \& electrolyte imbalance) .

- Eye lesions: -Corneal ulceration $\rightarrow$ scarring \& blindness.

- Urogenital :-In male (urethritis \&balanitis) in female,( vaginal ulcers )

\section{Diagnosis}

- History

-1-Minor type, sudden onset of vesiculo-bullous lesions develop rapidly (1-2days) .

- 2- Major type ,sudden onset fever vesiculo- bullous lesions develop rapidly(1-2 days).

- 3- recurrence

\section{Clinical examination}

-1-Bloody crusted appearance is fairly typical.

-2-Skin lesions

-3-Special Investigation

- Histopathologic features are nonspecific; intracellular edema

C.T. \& sub epithelial vesicles.

\section{Differential diagnosis:}

Electron Microscope :- should be differentiated from

Mucous Membrane Pemphigoid\&Pemphigus Vagarious; Bullous lichen planus; herpetic stomatitis, stomatitis medicaments

\section{Treatment:}

Self-limiting; supportive\& directed toward eliminationof;

A-Lesions: by topical steroid

B-Pain: - by topical anesthesia

C-Infection; by tetracycline mouth rinse

\section{7-B- Behcet's Syndrome}

- It is a multi-system
- (CVS, GIT, CNS, Pulmonary, Articular, Skin) inflammatory disorder of unknown etiology.

- Oral aphthous-like ulcers

-1- Genital lesion ;Recurrent genital ulceration

- Scrotum \& penis in males - labia in females

-2-Eye lesions

- Retinal vasculitis- conjunctivitis

3-Skin lesions

- Limbs , trunk \& around genitals

- A-Erythema nodosum B-Acneiform lesions

- C- Large pustule lesions d-Positive pathergy test

\section{7-C- Reiter's Syndrome}

- It is a triad consisting of urethritis

- (precedes other lesions ) arthritis\&conjunctivitis

- Age :- 30-40 years

- Sex :- common in females \& children

- Etiology :- unknown

- Clinical features:-

- Arthritis - conjunctivitis-skin lesions ; ( (macules or papules on palms \& sole )

- Oral lesions

- Buccal mucosa ,lips \& gingival lesions ;

- Painless,red,macules white raised border

- May ulcerate to produce painless aphthouslike ulcers.

- Palatal lesions :

- Small, red, purpuricspots .

- Tongue lesions ;

- geographic tongue

Treatment

- It is a self limiting disease of few month

- anti-inflammatory - analgesic -antibiotic- \& steroid

\section{Dedication:}

To Soul the oral medicine godfather \& my mentor Prof. Sami Sadek.

\section{References}

1. Sami Sadek. Oral Medicine for dental practitioners. 1998.

2. Burket's Oral Medicine 12th Edition. 2017 